ESAIM: M2AN

Vol. 41, No 2, 2007, pp. 427-445

DOI: $10.1051 / \mathrm{m} 2 \mathrm{an}: 2007024$
ESAIM: Mathematical Modelling and Numerical Analysis

www.edpsciences.org/m2an

\title{
MULTISCALE MATERIALS MODELLING: CASE STUDIES AT THE ATOMISTIC AND ELECTRONIC STRUCTURE LEVELS*
}

\author{
Emilio Silva ${ }^{1}$, Clemens Först $^{2}, \mathrm{Ju} \mathrm{Li}^{3}, \mathrm{Xi} \mathrm{Lin}^{2}$, Ting Zhu ${ }^{4}$ And Sidney Yip ${ }^{2}$
}

\begin{abstract}
Although the intellectual merits of computational modelling across various length and time scales are generally well accepted, good illustrative examples are often lacking. One way to begin appreciating the benefits of the multiscale approach is to first gain experience in probing complex physical phenomena at one scale at a time. Here we discuss materials modelling at two characteristic scales separately, the atomistic level where interactions are specified through classical potentials and the electronic level where interactions are treated quantum mechanically. The former is generally sufficient for dealing with mechanical deformation at large strain, whereas the latter is necessary for treating chemical reactions or electronic transport. We will discuss simulations of defect nucleation using molecular dynamics, the study of water-silica reactions using a tight-binding approach, the design of a semiconductor-oxide interface using density functional theory, and the analysis of conjugated polymer in molecular actuation using Hartree-Fock calculations. The diversity of the problems discussed notwithstanding, our intent is to lay the groundwork for future problems in materials research, a few will be mentioned, where modelling at the electronic and atomistic scales are needed in an integrated fashion. It is in these problems that the full potential of multiscale modelling can be realized.
\end{abstract}

Mathematics Subject Classification. 74A25, 74A45, 74F25, 74M05, 81V55.

Received: November 3, 2005.

\section{INTRODUCTION}

Currently there is widespread interest in identifying fundamental problems in multiscale materials modelling which combine scientific challenge with technological relevance. To provide a basis for such considerations, we revisit several of our recent investigations that address two current themes, the mining of atomistic simulations to extract insight into mechanical failure of materials, and the manipulation of electronic structure effects in nanomaterials innovation. Through these examples we hope to illustrate the fundamental capabilities of modelling and simulation for probing materials phenomena across different length and time scales. Five specific problems

Keywords and phrases. Multiscale modelling and simulation, fracture, molecular actuator, semiconductor interface.

* This work is supported by the National Science Foundation under grants ITR-020541, DMR-0325553, and IMR-0414849,

Honda $R \& D$, and the OSU Transportation Research Endowment Program.

1 Department of Civil and Environmental Engineering, MIT, USA.

2 Department of Nuclear Science and Engineering and Department of Materials Science and Engineering, MIT, USA.

syip@mit.edu

3 Department of Materials Science and Engineering, Ohio State University, USA.

4 Department of Mechanical Engineering, Georgia Institute of Technology, USA. 
will be discussed in Sections 1 and 2, starting with the question of how materials strength and deformation can be investigated at the atomistic level in terms of concepts of structural instability and the dynamical response to critical loading in nano-indentation. Then we consider the use of reaction pathway sampling to study how a crack front advances in ductile and brittle fracture. The same technique is used again in modelling the hydrolysis reaction between a silica nanorod and a water molecule, where the tight-binding approximation, a low-level quantum chemical method, is applied to treat the interaction. In the last two problems the electronic degrees of freedom are taken into account by applying density functional theory and Hartree-Fock calculations, one dealing with designing an oxide layer as interface with silicon in semiconductor devices, and the other concerning the use of electron-phonon coupling effects in conjugated polymers for molecular actuation.

This diverse sample of applications shows how different materials research problems require distinct levels of modelling capability and accuracy. Each problem plays a role toward contributing the groundwork for future studies where modelling at the electronic and atomistic scales are needed in an integrated fashion. In Section 3, we outline some of the major challenges in integrating different modelling and simulation levels in a true multiscale framework. To realize the benefits of multidisciplinary participation it is important that applied mathematicians, materials researchers and computer scientists are all involved.

\section{Mining atomistic Simulations: meChanical BeHAVior AND ENVIRONMENTAL EFFECTS}

\subsection{Theoretical strength of materials}

The theoretical basis for describing the mechanical stability of a crystal lattice lies in the formulation of stability conditions which specify the critical level of external stress that the system can withstand. Lattice stability is one of the most central issues in elasticity; it is fundamental to understanding structural transformations in solids, such as polymorphism, amorphization, fracture or melting. Born $[4,5]$ has shown that by expanding the internal energy of a crystal in a power series in the strain and requiring positivity of the strain energy, one obtains a set of conditions on the elastic constants of the crystals that must be satisfied to maintain structural stability. Born's original derivation considered the case of no external stress, the results could be applied to thermoelastic behavior such as melting at zero pressure. To discuss stress-driven instability one would need to consider the elastic stiffness coefficients [53]

$$
B_{i j k l}=C_{i j k l}+\Lambda_{i j k l}
$$

where $C_{i j k l}$ is the elastic constant tensor, and

$$
\Lambda_{i j k l}=\frac{1}{2}\left(\delta_{i k} \tau_{j l}+\delta_{j k} \tau_{i l}+\delta_{i l} \tau_{j k}+\delta_{j l} \tau_{i k}-2 \delta_{k l} \tau_{i j}\right)
$$

with $\delta_{i j}$ being the Kronecker delta symbol and $\tau_{i j}$ being the applied stress tensor. The general condition for the loss of stability is [54]

$$
\operatorname{det}|A|=0
$$

where $A_{i j k l}=\frac{1}{2}\left(B_{i j k l}+B_{k l i j}\right)$. In the absence of an external stress the elastic constants, which are intrinsic properties of the material, are sufficient to determine lattice stability, as equation (3) then becomes the Born criteria. Conversely, one can say that at finite stress material strength is no longer an intrinsic property of a material. The origin of the second term in equation (1) is seen to be the work done on the system by the external stress. In the case of a cubic crystal under hydrostatic loading, the stability conditions are particularly simple,

$$
C_{11}+2 C_{12}+P>0, \quad C_{11}-C_{12}-2 P>0, \quad C_{44}-P>0
$$

where $P<0$ ( $>0$ ) denotes compression (tension). The first condition in (4) defines stability in cohesion (nonvanishing bulk modulus), while the second and third inequalities imply positive tetragonal and rhombohedral 


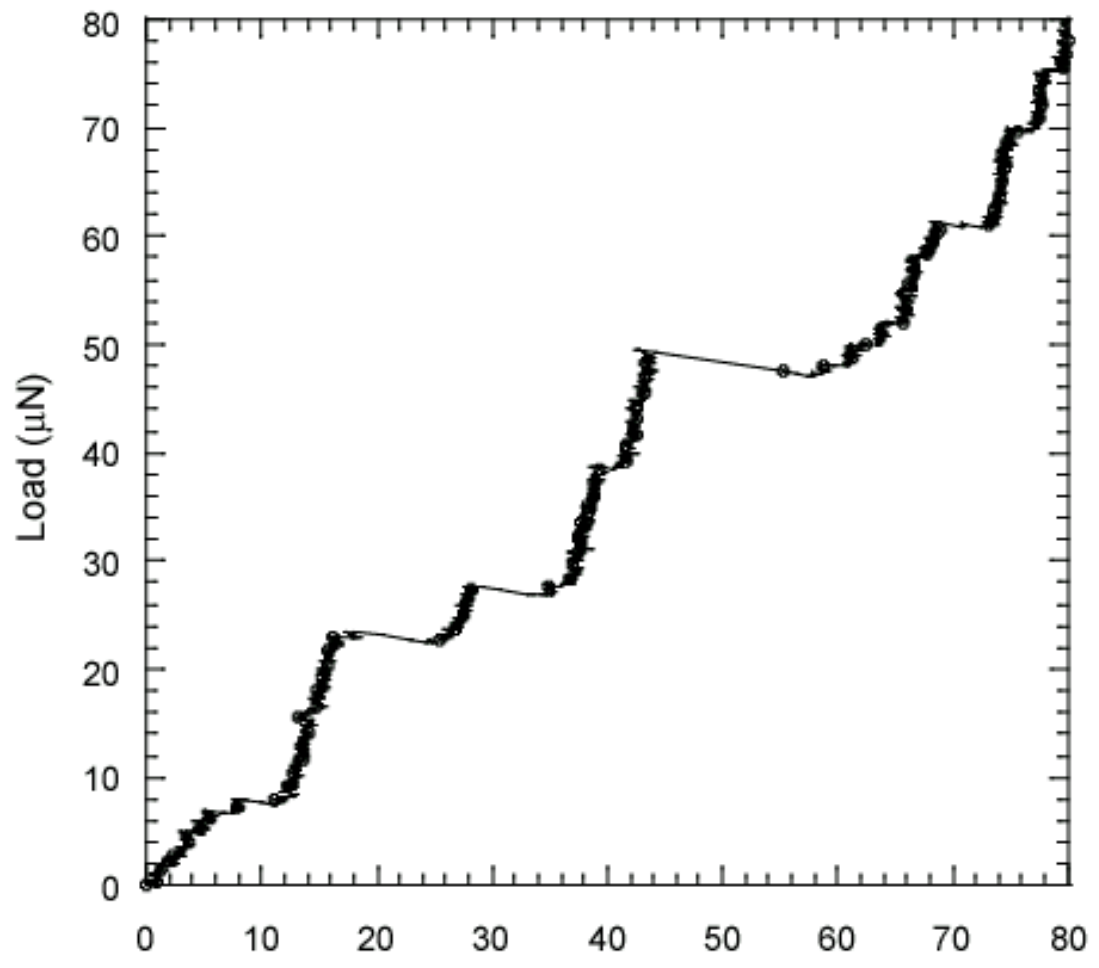

Figure 1. Typical load-displacement curve in a nano-indentation measurement (load control) [19]. Continuous increase of indentation depth with indenter force shows elastic deformation interspersed with horizontal jumps. These "bursts" are interpreted as the onset of dislocation activity, each jump corresponding to a local instability somewhere in the system (not necessarily immediately under the indenter).

shear moduli respectively (shear instability). These rather simple criteria have been helpful in elucidating simulation results on structural phase stability $[28,54]$.

Further discussions of the stability criteria may be found in the literature $[40,54,55,59]$. In the following we confine our attention to intrinsic or ideal behavior of homogeneous deformation of a defect-free the crystal, conditions achievable only in simulation.

\subsection{Instability in nano-indentation}

We consider the specific case of observing dislocation nucleation in a nano-indentation experiment as shown in Figure 1 [19]. The variation of the compressive stress with depth of indentation shows a continuous increase of depth versus load, as expected in an elastic deformation, interrupted by intermittent discontinuous jumps which indicate loss of local structural stability. These jumps have been interpreted as onsets of plastic deformation, associated with dislocation nucleation and multiplication events.

The elastic stability criteria (4) apply to a perfect crystal under homogeneous deformation. For inhomogeneous deformation such as the case of nano-indentation, one expects local defects to be nucleated at certain sites in the system (the weak spots) when the system is driven to a saddle point. A continuum-level description of homogeneous nucleation was first explored by Hill [21] in the concept of discontinuity of "acceleration waves". Later Rice [45] treated shear localization in much the same spirit and derived a formal criterion characterized by a tensor $\mathbf{L}$ playing the same role as the stiffness tensor $\mathbf{B}$. This formalism can be taken to the discrete-particle level to obtain a spatially-dependent nucleation criterion for practical implementation $[27,52,60]$. Consider a 
representative volume element (RVE) undergoing homogeneous deformation at finite strain to a current configuration $\mathbf{x}$. Expanding the free energy $F$ to second order in incremental displacement $\mathbf{u}(\mathbf{x})$, one obtains

$$
\Delta F=\frac{1}{2} \int_{V(\mathbf{x})} D_{i j k l} u_{i, j}(\mathbf{x}) u_{k, l}(\mathbf{x}) \mathrm{d} V
$$

where $D_{i j k l}=C_{i j k l}+\tau_{j l} \delta_{i k}, \tau_{i j}$ being the internal (Cauchy) stress, and $u_{i, j} \equiv \partial u_{i}(\mathbf{x}) / \partial x_{j}$. Applying a plane wave perturbation, $u_{i}(\mathbf{x})=w_{i} \mathrm{e}^{i \mathbf{k} \cdot \mathbf{x}}$, one arrives at the stability condition for the RVE,

$$
\Lambda(\mathbf{w}, \mathbf{k})=\left(C_{i j k l} w_{i} w_{k}+\tau_{j l}\right) k_{j} k_{l}>0 .
$$

In contrast to the stiffness tensor $\mathbf{B}, \Lambda$ is site-dependent, while just as before the sign of $\Lambda$ reflects the concavity of $F$. If a pair of $\mathbf{w}, \mathbf{k}$ exists such that $\Lambda$ is negative, then homogeneity of this RVE cannot be maintained and defect singularities will form internally. In other words, the elastic stability of the RVE can be determined by minimizing $\Lambda$ with respect to the polarization vector $\mathbf{w}$ and the wave vector $\mathbf{k}$. The minimum value of $\Lambda, \Lambda_{\min }$, is therefore a measure of the local micro-stiffness; an instability is predicted at the spatial position where $\Lambda_{\min }$ vanishes. We regard equation (6) to be an energy-based criterion, and minimization of $\Lambda$ at a material point clearly reflects the local environment. Notice that the Helmholtz form of the free energy has been used rather than the Gibbs form. This is because we are applying the plane wave perturbation in a system with periodic boundary condition, so no external work is involved [28].

Equation (6) has been applied to analyze MD simulation results on nano-indentation[27,45]. The first few predictions of instability according to the $\Lambda$ criterion were found to correspond closely with jumps in the simulated load-displacement curve, as shown in Figure 2. Because the simulations were performed at increments of fixed strain (displacement control mode), discontinuities appear as vertical jumps in contrast to Figure 1.

\subsection{Probing crack front extension through reaction pathway sampling}

Is it possible to study how a sharp crack propagates in a crystal lattice without actually driving the crack front to the point of instability? By this we have in mind finding the pathways of a crack front while the lattice resistance against its displacement is still finite. Although many molecular dynamics (MD) simulations on crack tip propagation have been reported (e.g., see [7]), this particular question has not been addressed until recently. We have implemented a method of following crack front evolution which involves using reaction pathway sampling to probe the minimum-energy path (MEP) [23] for the crack front to advance by one atomic lattice spacing, while the imposed load on the system is below the critical threshold. Here we compare the results for a metal $(\mathrm{Cu})[60]$ and a semiconductor $(\mathrm{Si})$ [61] to illustrate how ductility or brittleness of the crystal lattice can manifest in the mechanics of crack-front deformation at the nanoscale.

We begin by applying a mode-I (uniaxial tensile) load in incremental steps to a 3D atomically straight crack front as shown in Figure 3a. Initially the crack would not move spontaneously because the driving force is not sufficient to overcome the intrinsic lattice resistance. Imagine a final configuration, a replica of the initial configuration with the crack front translated by an atomic lattice spacing in the direction of crack advancement. At low loads, e.g. $K_{\mathrm{I}}^{\prime}$ as shown in Figure 3b, the initial configuration (open circle) has a lower energy than the final configuration (closed circle). They are separated by an energy barrier which represents the intrinsic resistance of the lattice. As the loading increases, the crack will be driven toward the final configuration. One can regard the overall energy landscape as being tilted toward the final configuration with a corresponding reduction in the activation barrier, see Figure 3b. As the load increases further the biasing becomes stronger. As long as the barrier remains finite, the crack will not move out of its initial configuration without additional activation, such as from thermal fluctuations. When the loading reaches the point where the lattice-resistance barrier disappears altogether, the crack is then unstable at the initial configuration; it will move without any thermal activation. This is the athermal load threshold, denoted by $K_{\text {Iath }}$ in Figure $3 \mathrm{~b}$. In our simulation, we study the situation where the applied load is below this threshold, thereby avoiding the problem of a fast moving crack that is usually over-driven. 


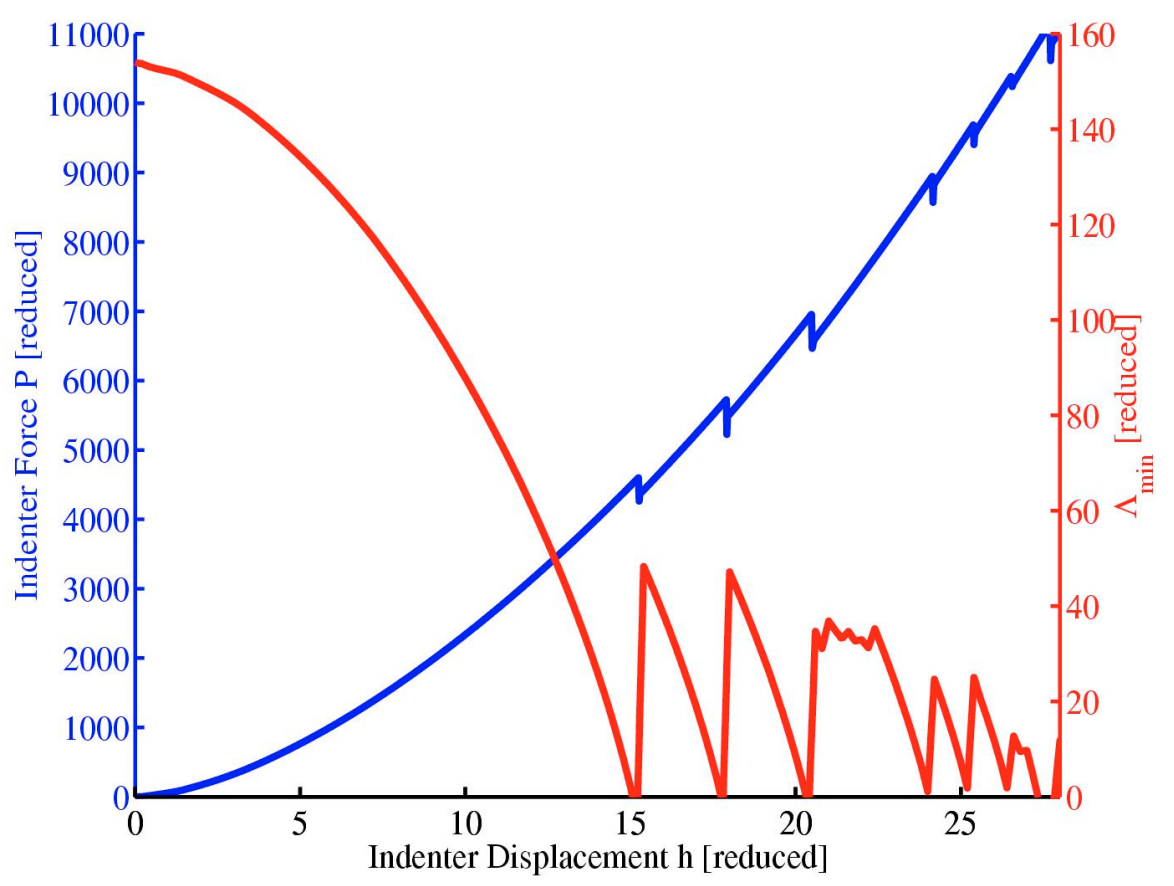

FIGURE 2. Load-displacement response in an MD simulation of nano-indentation (load control) on a slab of single crystal of $\mathrm{Cu}$ (rising curve), showing several vertical jumps corresponding to the dislocation bursts of Figure 1 [45]. The decreasing curve gives the variation of $\Lambda_{\min }$ with indenter displacement. According to equation (6), the vanishing of $\Lambda_{\text {min }}$ would signal a structural instability.

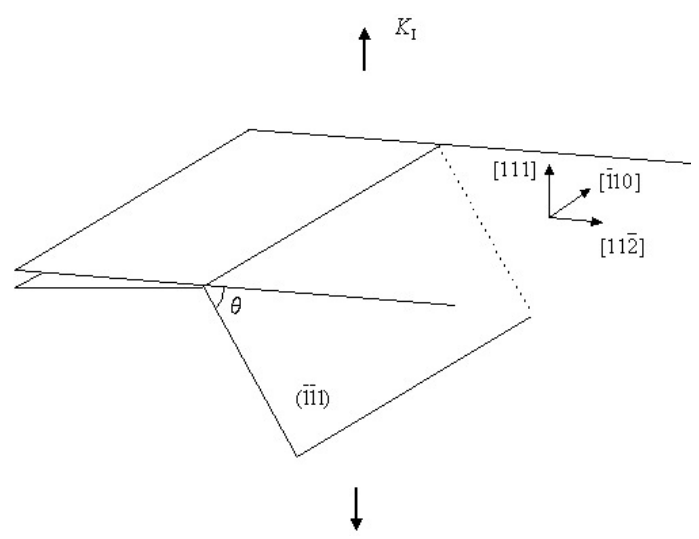

(a)

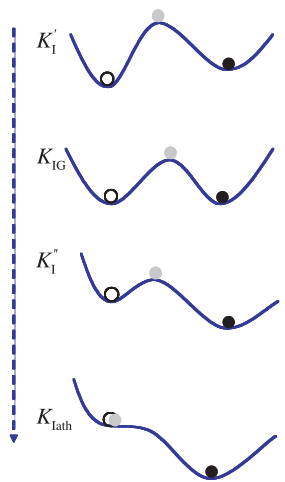

(b)

Figure 3. (a) Schematics of a 3D atomically sharp crack front under a mode-I load $K_{\mathrm{I}}$; (b) energy landscape of the crack system at different loads $\left(K_{\mathrm{I}}^{\prime}<K_{\mathrm{IG}}<K_{\mathrm{I}}^{\prime \prime}<K_{\text {Iath }}\right)$. Open circle represents the initial state of a straight crack front under an applied load $K_{\mathrm{I}}$, closed circle is the final state after the crack front uniformly advances by one atomic spacing (under the same load $K_{\mathrm{I}}$ as the initial state), and shaded circle corresponds to the saddle-point state in between. 


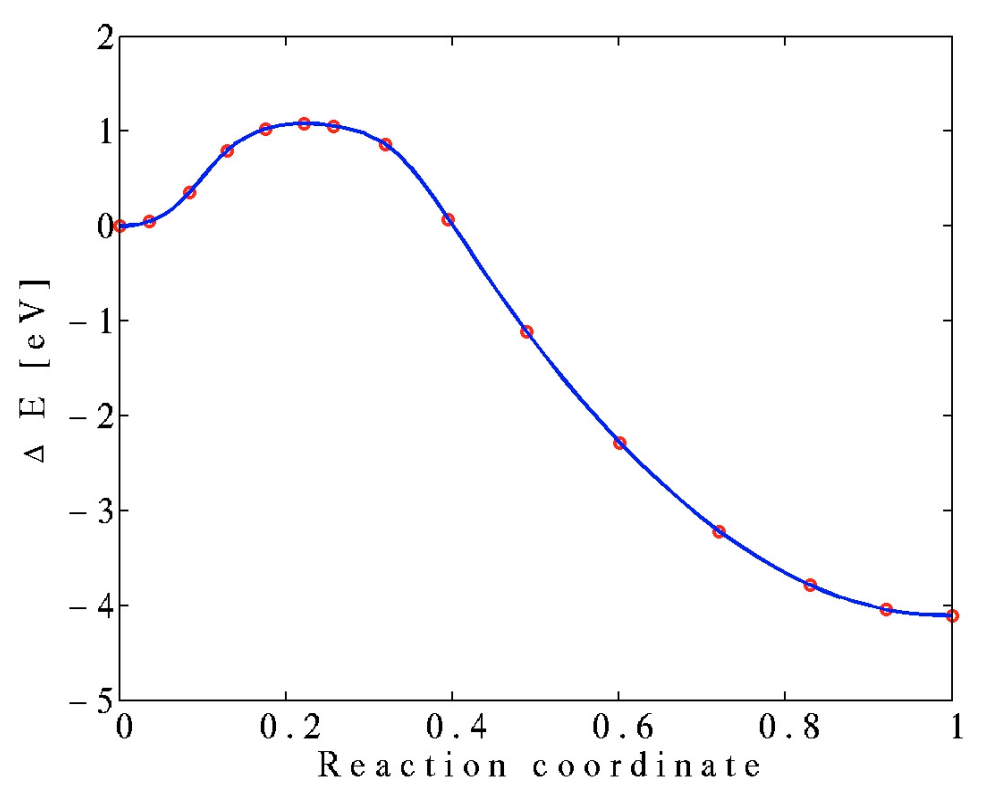

Figure 4. MEP of dislocation loop emission in $\mathrm{Cu}$ at a load of $G=0.75 G_{\text {emit }}$.

The cracks in $\mathrm{Cu}$ and $\mathrm{Si}$ that we will compare are both semi-infinite cracks in a single crystal, with the crack front lying on a (111) plane and running along the [110] direction. The simulation cells consist of a cracked cylinder cut from the crack tip, with a radius of $80 \AA$. The atoms located within $5 \AA$ of the outer surface are fixed according to a prescribed boundary condition, while all the remaining atoms are free to move. To probe the detailed deformation of the crack front, the simulation cell along the cylinder is taken to be as long as computationally feasible, $24(\mathrm{Cu})$ and $20(\mathrm{Si})$ unit cells. Periodic boundary condition is imposed along this direction. For interatomic potentials we use a many-body potential of the embedded atom method type for $\mathrm{Cu}$ [39], and a well-known three-body potential model proposed by Stillinger and Weber (SW) for Si [49].

Next, we compare studies of $\mathrm{Cu}[60]$ and $\mathrm{Si}$ [61] in terms of MEP profiles and atomic displacement distributions. Figure 4 (blue line) shows the MEP for the nucleation of a dislocation loop from the crack front in $\mathrm{Cu}$, loaded at $K_{\mathrm{I}}=0.87 K_{\text {Iath }}$. Notice that at this loading the final state is strongly favored over the initial state. Figure 4 shows clearly the presence of a lattice-resistance barrier at the particular loading. To visualize the atomic configurations along the MEP, we turn to displacement distributions along the crack front. Figure 5 shows the saddle-point configuration of a partial dislocation loop along the crack front, the loop being delineated by atoms which have coordination number different from that of atoms in the bulk crystal. These are the atoms in the dislocation core. Figure 6a is a contour plot of the shear displacement distribution.

One can see clearly the shape of a dislocation loop bowing out. It is evident that the distribution represents the development of an embryonic dislocation loop. The profile of $b / 2$ shear displacement is a reasonable representation of the locus of dislocation core, slips between atom pairs across the slip plane of one $b$. Also this is an indication that the enclosed portion of the crack front has been swept by a fully formed dislocation. Besides shear displacement, normal, or opening displacement, in the direction along $x_{3}$, [111], is also of interest. The corresponding distribution is shown in Figure 6b. One sees that large displacements are not at the center of the crack front. In Figures $6 \mathrm{a}$ and $6 \mathrm{~b}$ we have a detailed visualization of the crack front evolution in three dimensions. The largest displacements are indeed along the crack front but they are not of the same character; the atoms move in a shear mode in the central region and in an opening mode on the two sides.

Figure 7 shows the opening displacement distribution in $\mathrm{Si}$ at the Griffith load $K_{\mathrm{IG}}=0.646 \mathrm{MPa} \sqrt{\mathrm{m}}$. We see a new feature in the outline of displacements of intermediate magnitude (blue line). In the region ahead of the crack front the distribution of these displacements has the shape of a rectangular wedge protruding in 


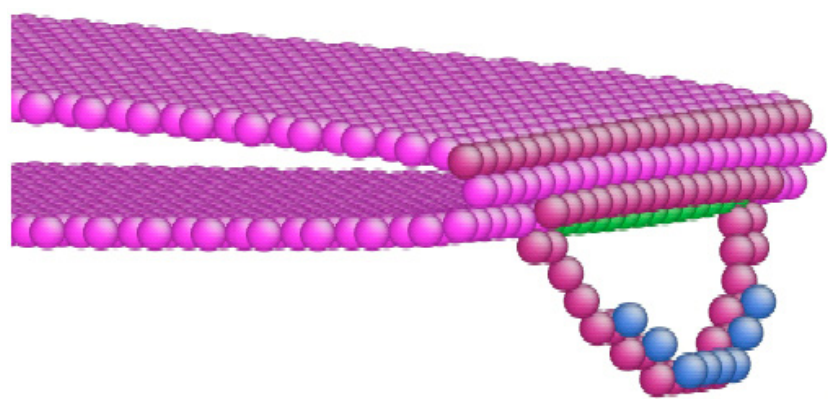

FigurE 5. Atomic structure of the saddle-point configuration of dislocation loop nucleation.

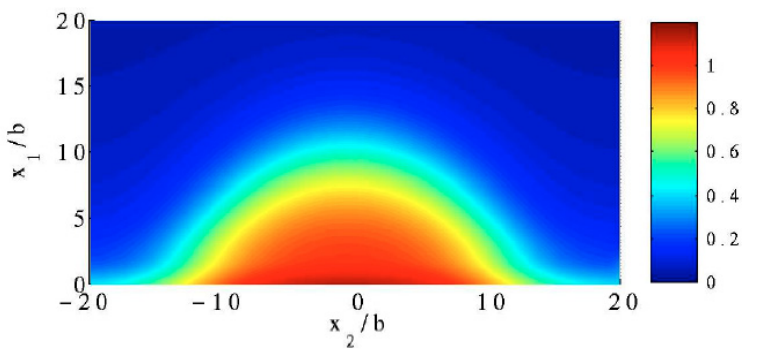

(a)

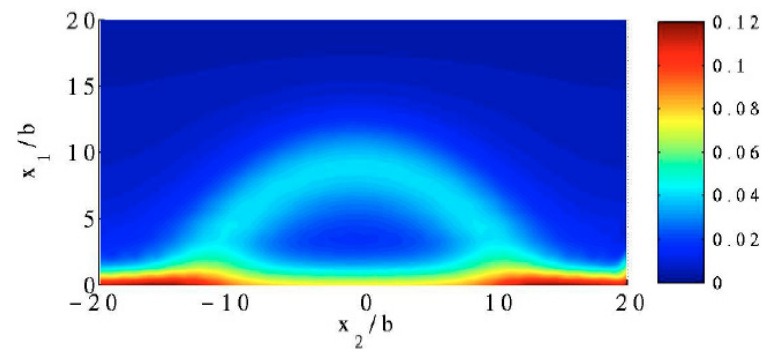

(b)

Figure 6. Contour of (a) shear displacement (normalized by $b=1.476 \AA$ ) and (b) opening displacement (normalized by the interplanar-spacing $h=2.087 \AA$ ) across the slip plane at the saddle-point state.

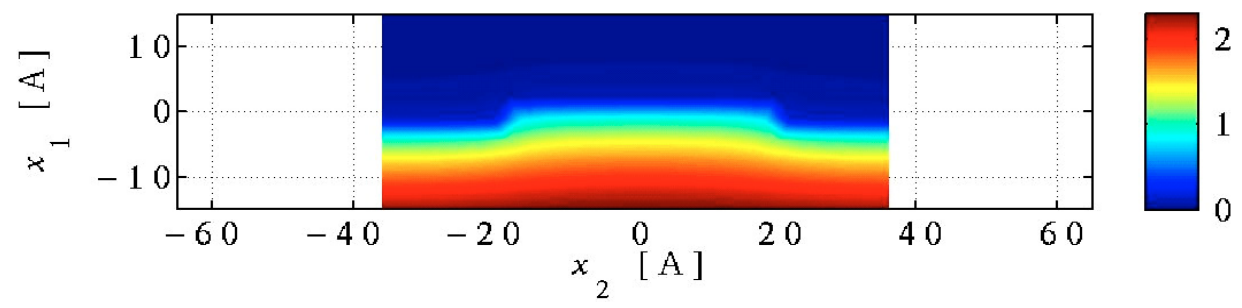

FIGURE 7. Contour of opening displacement distribution at the Griffith load $K_{\mathrm{IG}}$.

the direction of crack front advancement. The presence of a wedge shape suggests a kink mechanism of crack advancement, namely, nucleation of a local kink distortion followed by spreading across the entire crack front. It is significant that this behavior is not seen in Figure $6 \mathrm{~b}$. Taking $\mathrm{Cu}$ to be a prototypical ductile material, we see that while the crack opening still occurs at the front, the large normal displacements lie outside the central region enclosed by the emerging dislocation loop. We attribute this feature to a mode-switching, or sheartension coupling, process. The initially large opening displacements in the region swept by the emerging loop are relaxed by giving way to large shear displacements, which are then carried away by the emitted dislocation loop.

It is relevant to interpret the behavior of atomic displacements at the transition state in $\mathrm{Cu}$ and $\mathrm{Si}$ on the basis of the nature of interatomic bonding in these two materials. One expects that the crack front response in $\mathrm{Cu}$ should reflect delocalized metallic bonding while that in Si should correspond to directional, localized 


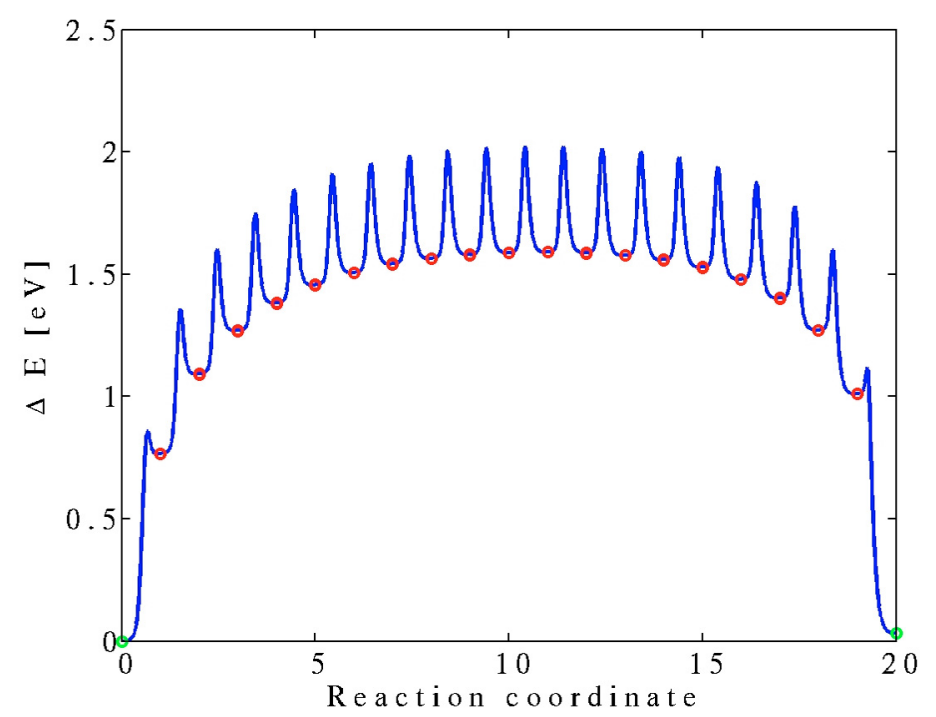

FiguRE 8. MEP of crack extension in Si at the Griffith load $K_{\mathrm{IG}}=0.646 \mathrm{MPa} \sqrt{\mathrm{m}}$.

covalent bonding. In terms of characteristic features of the energy landscape along the reaction path, we see in $\mathrm{Cu}$ a rather smooth MEP with a single major nucleation barrier, indicating that crack advancement involves a concerted motion of atoms to overcome this barrier by thermal activation. In contrast MEP in Si reveals the existence of significant secondary barriers (cusps in Fig. 8) which should be a general feature of covalently bonded crystals. In this case crack extension proceeds via individual bond breakings, a series of thermally activated events of kink-pair formation and lateral kink migration along the front. It is of interest to point out the crack front mobility is not only controlled by kinks at the atomic size scale as demonstrated in this work, acoustic emission and fractographic measurements have indicated the crack advancement at the mesoscopic scale is also governed by the kink mechanism which involves a process of unzipping along the crack front (W.W. Gerberich, private communication [29]).

The fact that a kink mechanism appears to play a central role in crack front mobility raises an interesting question of the implications of structural similarity between the crack front, acting as the core of a sharp crack, and the core of a dislocation, both being "line defects" in a crystal lattice. It is rather well known that dislocation mobility in a directionally bonded crystal like $\mathrm{Si}$ is governed by thermal activation of nucleation and migration of kink pairs [8]. The present results showing that a similar mechanism also operates in crack front advancement reinforces the notion that mobility fundamentally depends on crystal structure and chemical bonding. From this perspective the appearance of kink-like structure in Figure 7 is perhaps to be expected. Since dislocation mobility and crack-tip extension are both active topics for modelling and simulation, recognition of their underlying connections could lead to a broader appreciation of the role of structure and bonding [41] in controlling both phenomena.

\subsection{Water-silica reaction}

Chemical reaction rates in solids are known to depend on mechanical stress levels, an effect generally characterized through the variation of the activation energy barrier in the presence of stress. A typical phenomenon is the stress corrosion of silica glass by water; the strength of the glass decreases with time when subjected to a static load in an aqueous environment [56]. From a microscopic viewpoint [38] it is believed that the intrusive water molecules chemically attack the strained siloxane $(\mathrm{Si}-\mathrm{O}-\mathrm{Si})$ bonds at the crack tip, causing bond rupture and formation of terminal silanol $(\mathrm{Si}-\mathrm{OH})$ groups which repel each other at the conclusion of the process. 


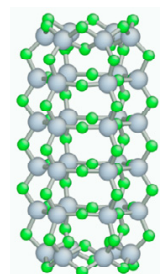

(a)

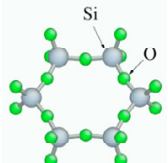

(b)

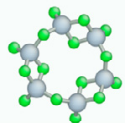

(c)

Figure 9. Structure of the silica nanorod. (a) Full nanorod, (b) corner-sharing internal ring, (c) edge-sharing end ring.
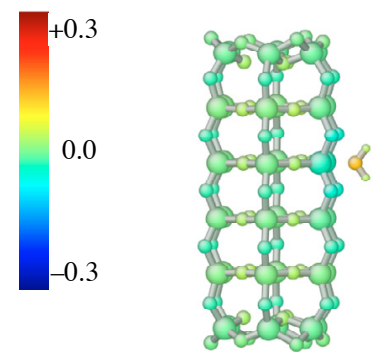

(a)

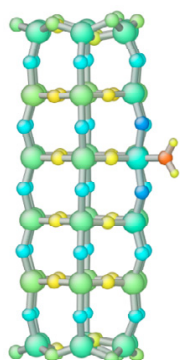

(b)

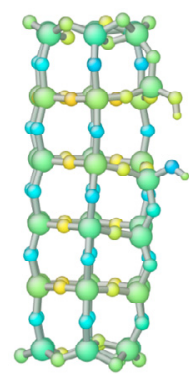

(c)

Figure 10. Nanorod deformation by direct simulation.

(a) Physisorption of water, (b) chemisorption of water, (c) hydrolysis of the nanorod. Atoms are color-coded according to the charge variation relative to the unstrained initial state.

Given the capability described above for visualizing atomic displacements (motions) in a nanometer-sized region, we can imagine the water-silica reaction can be studied using this "atomic-resolution microscope". Figure 9 shows a nanostructure of silica in the form of a rod composed of 108 atoms of Si and O, arranged as a stack of four rings of corner-sharing silica tetrahedra capped at the ends by two rings of edge-sharing tetrahedra. The resulting structure is a stoichiometric silica nanorod which is free of dangling bonds. The energy of the system was calculated using the semiempirical molecular orbital method PM5 [48].

To prepare the nanorod structure in a strained state we subject it to quasi-static tension. In each deformation step all the atomic positions are scaled to impose the desired strain. Then the silicon atoms in the two end rings are held fixed, while all the other atoms in the system are allowed to relax to minimize the energy. This procedure corresponds to a quasi-static strain controlled experiment at $0 \mathrm{~K}$.

Next a water molecule is brought to within $5 \AA$ of the nanorod, and full system relaxation is performed using PM5. Three distinct stages of reactive response are observed, as shown in Figure 10. The first is physisorption of the water molecule (Fig. 10a), which persists up to a critical strain level whereupon the oxygen in the water molecule chemically bonds to the nearest silicon atom (Fig. 10b), the bond length being the same as the $\mathrm{Si}-\mathrm{O}$ bonds in the nanorod. With further deformation the siloxane ( $\mathrm{Si}-\mathrm{O}-\mathrm{Si}$ ) bond is broken (Fig. 10c). At this point, the water molecule abruptly dissociates and two stable silanol ( $\mathrm{Si}-\mathrm{OH})$ groups are formed. The hydrolyzed nanorod is still able to continue its deformation until stress concentration causes eventual failure.

This sequence of responses, revealed by energy minimization, allows us to identify three distinct metastable steps in the water-assisted failure of the nanorod. Due to the quasi-static nature of the simulation, each stage occurs when the activation barrier vanishes. However, because these are metastable states, we are unable to observe directly the reaction pathway. 


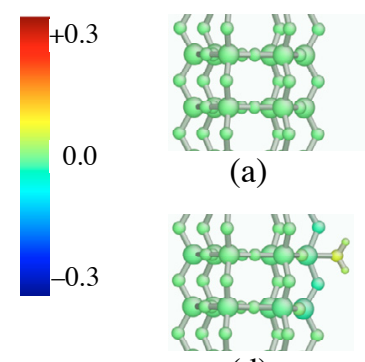

(d)

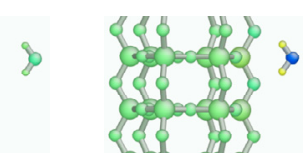

(b)

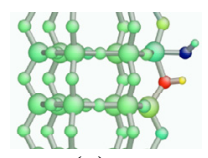

(e)

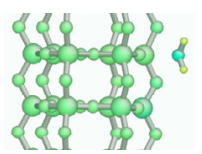

(c)

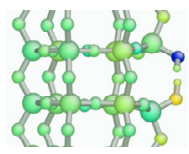

(f)

FiguRE 11. Water-silica nanorod reaction at reduced stress 0.3. (a) Initial step, (b) physisorption of the water molecule, (c) first saddle point, (d) metastable molecularly adsorbed state, (e) proton transfer at the second saddle-point and (f) hydrolyzed state.

As in the previous section, we use a method known as the Nudged Elastic Band (NEB) [23] to determine the minimum energy path (MEP) for the water-silica nanorod reaction, and thereby reveal the mechanisms and the corresponding activation barriers between the metastable states.

The minimum energy path for hydrolysis of the nanorod depends on the stress state, as seen in the simulations above. While keeping the strain constant, the NEB method allows us to induce the system to go over activation barriers toward the hydrolyzed state, simultaneously allowing us to observe the intermediate transition states. Figure 11 shows the main steps in the hydrolysis reaction at one third of the critical stress needed to hydrolyze the nanorod. Starting with a water molecule near the nanorod (Fig. 11a), we observe its movement toward the nanorod until it reaches the physisorbed state (Fig. 11b). Going over the first activation barrier, we can see the saddle-point state (Fig. 11c) before the molecularly adsorbed (chemisorbed) state (Fig. 11d). This is in contrast with the direct simulation, where we could not observe the intermediate state. Continuing in the reaction path, the system goes through another activation barrier, with a second saddle point (Fig. 11e). At this point, we can see the proton transfer from the water molecule to the neighboring oxygen atom in the siloxane bond that is being broken. This activated state was not accessible through the quasi-static simulation. Finally, the reaction ends at the hydrolyzed state (Fig. 11f), where the siloxane bond is broken and silanol groups are formed.

The energy profile for this minimum energy path is shown in Figure 12, which also displays the effect of stress level on the reaction. As the stress increases, the activation barriers are lowered and the hydrolyzed state becomes more favorable.

\section{Electronic Structure efFects in nanoscience}

\subsection{Computational design of semiconductor interfaces}

A particularly effective use of electronic structure techniques in multiscale modelling and simulation is the study of chemical reactions at materials interfaces. As a result of the continuing miniaturization of electronic devices, around a billion transistors are currently packed into one square centimeter, each being a composite of semiconducting, insulating, and metallic materials [22]. The presence of different types of bonding, from covalent to ionic to metallic, means that reliable simulation of the device structures, properties and performance will have to deal explicitly with electronic effects across interfaces between different materials.

Consider the problem of introducing transition metal oxides, so-called high- $k$ dielectrics, as the insulating layer between silicon and the metallic gate electrode. Because silicon and the oxides have fundamentally different bonding behavior (covalent vs. mostly ionic), it is a challenge to avoid having broken bonds at the interface which have a strong detrimental effect on the electronic properties. A way to solve this problem is to first understand the process of oxide growth on silicon through metal deposition and oxidation. Electronic structure calculations can be used to investigate how metal atoms can bond with silicon during deposition to form equilibrium structures at the surface. In this way electronic defects such as broken bonds can be identified. 


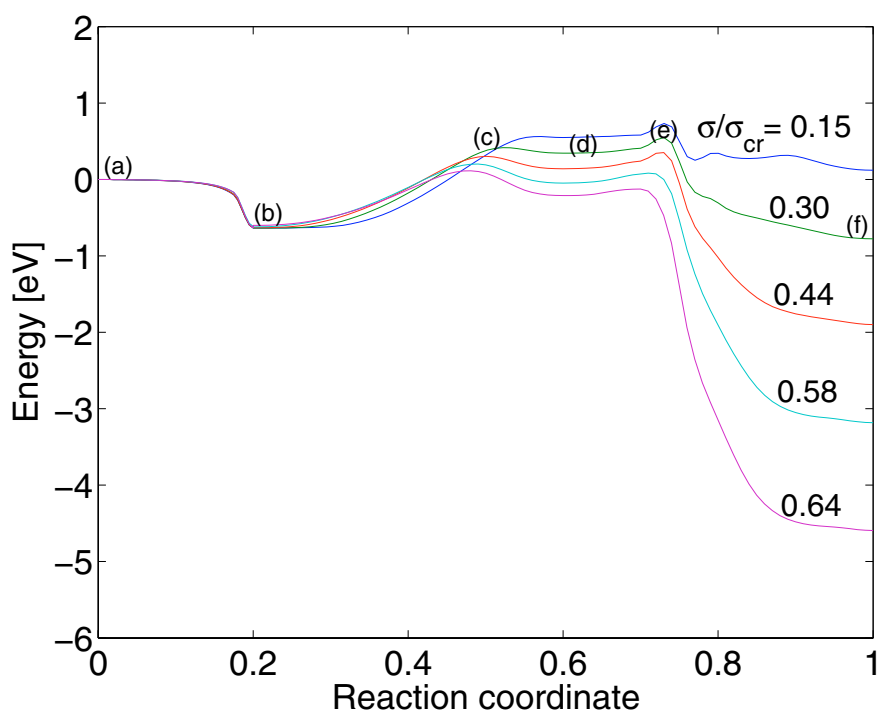

FiguRE 12. Relative energy in water-silica nanorod reaction. At reduced stress 0.3 , (a) initial state, (b) physisorbed state, (c) first saddle point, (d) metastable molecularly adsorbed state, (e) second saddle-point and (f) hydrolyzed configuration.

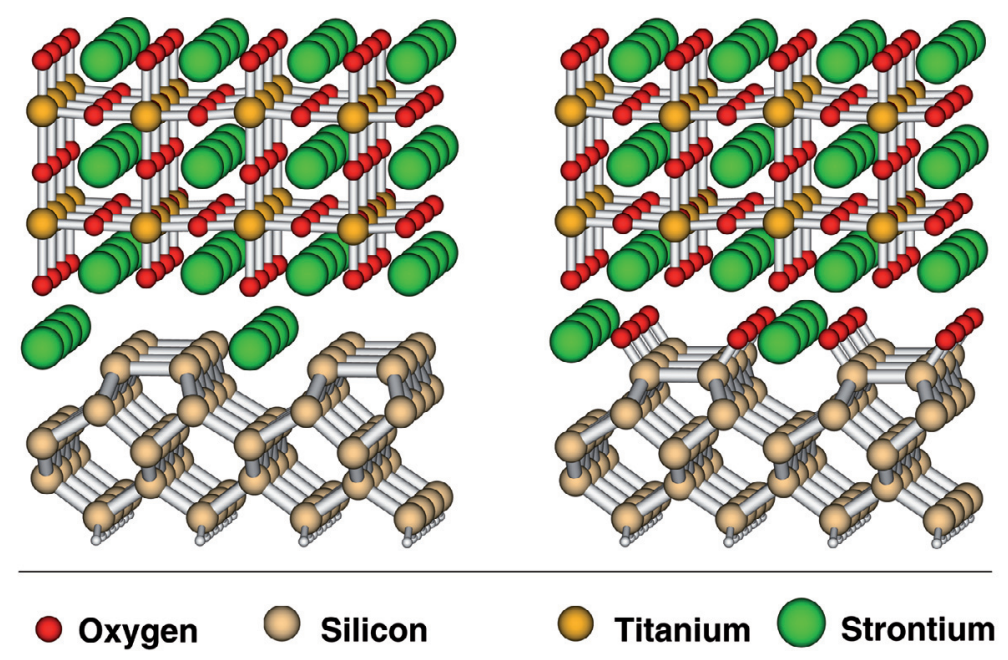

Figure 13. The two relevant interface structures between silicon and $\mathrm{SrTiO}_{3}$. At the bottom of each structure are the first five atomic layers of the silicon substrate, terminated by $1 / 2$ monolayer of strontium. On top we see crystalline $\mathrm{SrTiO}_{3}$.

The interface involving silicon $(\mathrm{Si})$ and strontium titanate $\left(\mathrm{SrTiO}_{3}\right)[37]$ is the example that has been recently studied $[2,16]$. It was found that a specific coverage of one half monolayer of $\mathrm{Sr}$ atoms will chemically saturate the silicon substrate (leaving no dangling bonds), as the left panel of Figure 13.

Besides ensuring no broken bonds at the interface, there is also the problem of alignment of the band structure (the electronic states) between the oxide and silicon. An offset of the conduction states acts as an electron injection barrier, resulting in an insulating behavior of the oxide layer. For many high-k oxides this offset is found to be too small. To solve this problem simulations have predicted that one can introduce a dipole 

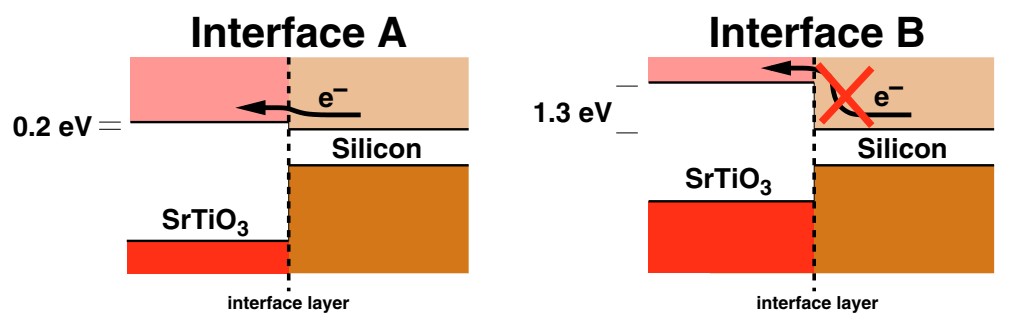

FigurE 14. Schematic representation of the band-offset between silicon and $\mathrm{SrTiO}_{3}$. In case of interface A there is only a negligibly small offset. As a result interface A does not exhibit sufficient insulating properties. The dipole introduced by the additional $\mathrm{O}$ atoms in interface $\mathrm{B}$ increases the offset to a value of $1.3 \mathrm{eV}$.

at the interface by selectively oxidizing the interfacial Si atoms (compare right panel of Fig. 13). This dipole shifts the electrostatic potential and thus also the band structure in the oxide and results in an acceptable value for the band offset. The effect is visualized in Figure 14.

Using this specific interface as an example we have demonstrated that the explicit calculation of the electronic structure of the interface is crucial to make viable predictions. Neither the chemically saturated interface nor the dependence of the insulating behavior on the stoichiometry at the interface could have been addressed using classical, interatomic potentials. Further discussions may be found in references $[2,16,17]$.

\subsection{Molecular actuation: mechanics driven by the quantum nature of electrons}

In the previous sections, we discussed simulation studies which involve (1) mechanical behavior that can be fully described by atomistics (homogeneous nucleation of the first dislocation); (2) crack front extension analyzed by a reaction pathway analysis, still involving only atomistics; (3) water-silica interactions and reactions treated with a combination of atomistics and semi-empirical quantum mechanics; and (4) the design of semiconductorsoxide interfaces which requires full electronic structure calculations. For the final topic in this perspective, we consider a problem where the nature of materials mechanics at the macroscale is governed by the quantum wave nature of electrons. We start from the theory that explains the fascinating electrical conductivity of conjugated polymers, show how the theory can be extended to describe fast mechanical deformations, and conclude with implications for biological macromolecules.

Scientific interests in low-dimension materials have grown rapidly since the feasibility of an organic superconductor at temperatures above room temperature was reported [33]. While superconductivity has not yet been realized, precise controls of doping levels allow macroscopic films of quasi-one-dimensional conjugated polymers at room temperatures to cover virtually the whole conductivity spectrum of all known materials, from insulators such as diamond $\left(10^{-14} \mathrm{~S} \cdot \mathrm{cm}^{-1}\right)$ to conductors such as copper $\left(10^{5} \mathrm{~S} \cdot \mathrm{cm}^{-1}\right)[36,46]$. That this conductivity range is possible is due to the creation of highly mobile charge carriers called solitons [20], which are strongly coupled electrons and phonons.

In three-dimensional metals, there is no band gap within the partially filled conduction band. Therefore, electrons of Fermi energy $\left(\varepsilon_{F}\right.$ in Fig. 15a) can move freely among occupied and unoccupied states that are continuous in energy. In one-dimensional metals, however, free Fermi electrons are trapped due to strong scattering by phonons of the same Fermi frequency, for example $\pi /(2 a)$ in the half filled band as illustrated in Figure 15, where $a$ is the lattice constant of the 1D crystal. The phonon trapping of Fermi electrons is inevitable in one-dimension because for any infinitesimal lattice distortion $u$, electronic energy decrease, which is proportional to $u^{2} \ln u$, dominates over the increase of lattice elastic energy increase, which is proportional to $u^{2}$. The net effect is to lower the energies of the occupied states and raise the energies of unoccupied states around the Fermi level (Fig. 15b), thereby opening a gap $\Delta$ at the Fermi level (Fig. 15c), with $\Delta$ proportional to $u$. This means that conventional metals do not exist in one-dimension, a result known as the Peierls distortion [43]. 


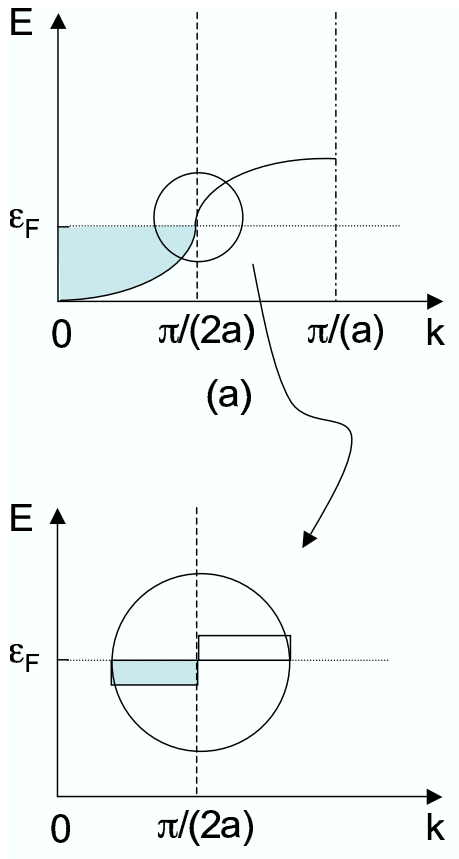

(b)

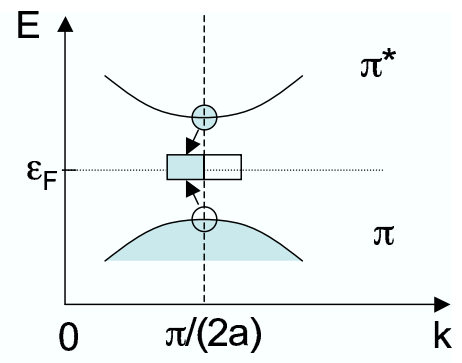

(d)

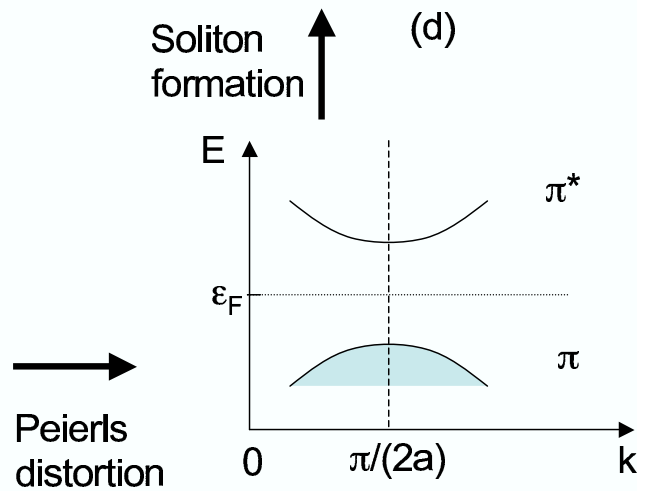

(c)

Figure 15. One-dimensional Peierls distortion of the half filled band and soliton formation in the gap. Strong coupling between phonons and electrons at the Fermi surface (a) and (b) opens up a finite band gap (c) at the Fermi energy. Upon creation, electrons and holes are self-directed to energetically more stable states in the gap via strong couplings to phonons, forming electron and hole solitons $(d)$.

For the chain trans-polyacetylene, $t$-PA, $-(-\mathrm{CH}-)_{n^{-}}$, the prototype of conjugated polymer, the band gap $\Delta$ is about $1.5 \mathrm{eV}$.

The beauty of the physics of one-dimensional systems follows immediately after the creation of electrons (or holes) in the Peierls distorted structure. Upon photo-excitation or chemical doping, holes (similarly for electrons) will be injected into the occupied states (shaded in Fig. 15) below the Fermi energy. In conventional solids, this newly added hole/electron is completely localized at low temperatures because of the finite gap. We label it as the maximally localized state in Figure 16. Having an energy that differs from $\varepsilon_{F}$ by only one half of the small band gap $\Delta(\sim 1.5 \mathrm{eV})$, this state is still subject to strong electron-phonon coupling. Therefore, lattice is distorted again to lower the electronic energy against a penalty of increased lattice elastic energy. A soliton is formed when the energy lowering and increase are balanced. Additionally, the induced lattice distortion causes the charge carried by the hole/electron to be a partially localized state (Fig. 16).

Because it is coupled to the phonons, the hole is neither a delocalized electron as in metals, nor a tightlybound electron as in insulators. In $\pi$-conjugated polymers, such a phonon-dressed hole can be highly mobile along the 1D conjugated carbon backbone because of its localized distribution. It is estimated by semi-empirical arguments that the soliton contains a half-width of $7 \mathrm{CH}$ sites in $t$-PA [50]. Our ab initio quantum mechanics computations indicate a similar spatial distribution of the soliton wavefunction, as shown in Figure 17 [30]. The nodal structures (zero possibility of finding electrons locally) between the alternating + (red lobes) and - (green lobes) phases indicate the charge density wave nature of solitons. From transition state sampling methods, we estimated the energy costs to move a soliton in the Peierls distorted 1D carbon backbone is equivalent to $10^{-13} \mathrm{~K}$, three orders of magnitude below the lowest temperatures ever achieved in laboratory [26]. Moreover, 


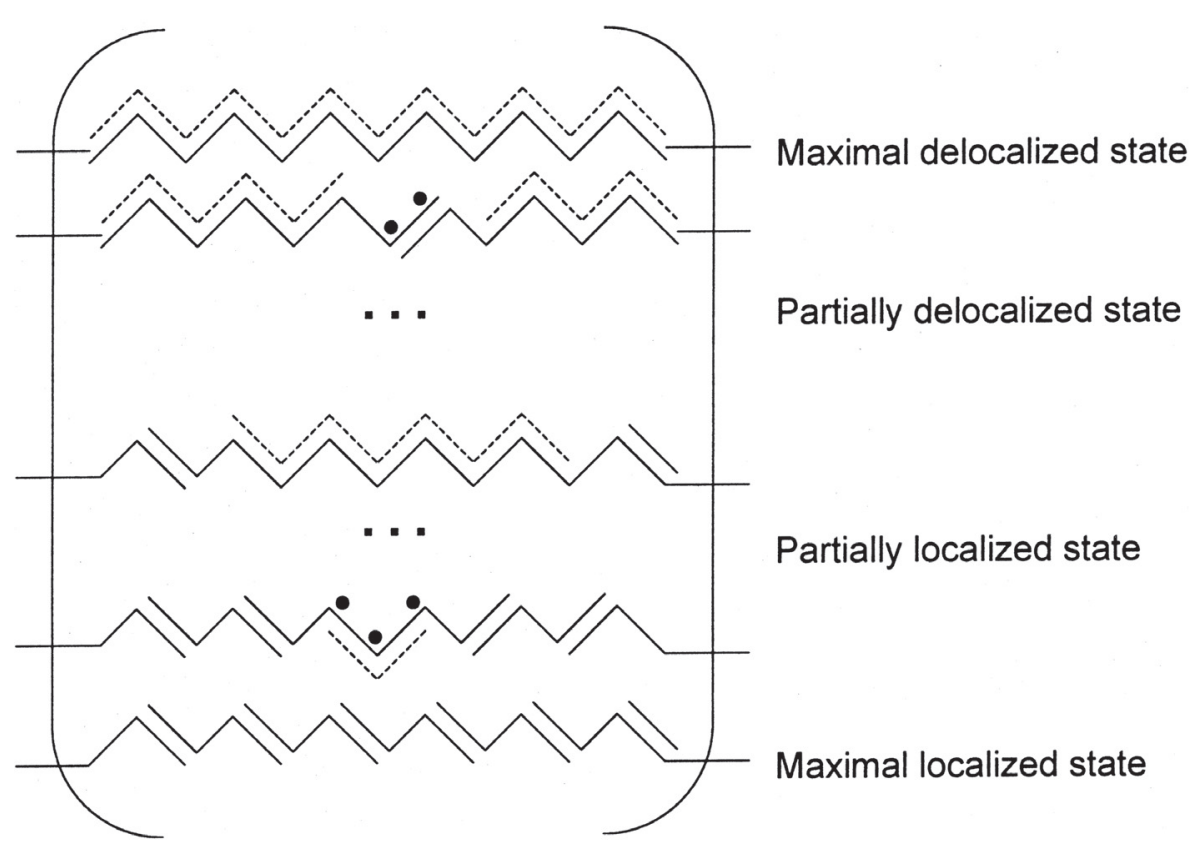

FigURE 16. Schematic representation of electron spatial distributions of different localization states in $t$-PA: maximal delocalized state for the homogeneous metallic state (Fig. 15a), maximal localized state for the Peierls distorted state (Fig. 15b and Fig. 15c). Formation of the soliton state (Fig. 15d) can be viewed as compensation between the metallic delocalization and Peierls localization, equilibrated at a minimum energy cost.

the effective mass of solitons is estimated to be roughly the same order as electrons [32,50]. The negligible barrier height and small effective mass make solitons the ideal charge carriers for $1 \mathrm{D}$ systems.

Despite the fact that the essential physics lies in 1D, we find soliton to behavior to be even more remarkable when the $3 \mathrm{D}$ nature of the conjugated polymer chains is taken into account. Besides the $\mathrm{C}-\mathrm{C}$ stretching modes, the dynamics now involves $\mathrm{C}-\mathrm{C}-\mathrm{C}$ bending and $\mathrm{C}-\mathrm{C}-\mathrm{C}-\mathrm{C}$ torsional modes. The original soliton theory can be extended to include these new modes via an extended Hubbard model [30]. By coupling to these new degrees of freedom, solitons play a central role in controlling the bending and torsional conformations in trans-and cis-polyacetylene, respectively. In polypyrrole, which is used in many practical applications, soliton-controlled conformations can reversibly switch the polymer chain between a helical reduced state and a localized straight state (Fig. 18). Accompanying the conformation changes, significant strain and stress are generated at a high speed $\left(\sim 10^{3} \mathrm{~m} / \mathrm{s}\right)$, which could lead to a new generation of molecular actuators $[30,31,58]$.

Following similar ideas, efforts have been made to understand the electrical conductance of DNA [1,15], which also contains helical structures. Figure 19 shows the wavefunction of a polaron, the soliton-anti-soliton pair, in poly(dA)-poly(dT) DNA molecule. The existence of small polaron in the conjugated $\pi$ stacks of DNA is a necessary condition to achieve a high level conductance, because $1 \mathrm{D}$ conventional metal does not exist and DNA backbone is completely saturated. The significance of having highly conducting DNA is two fold. From the biology side, charge injections are often associated with damage, mutation, and repair processes in DNA; from the materials side, conducting DNA wires are building blocks for nanoscale quantum computation devices.

\section{The Challenge of Multiscale materials MODElling}

We have discussed several problems, using our own studies as examples, where atomistic simulation and electronic structure calculations lead to a better understanding of materials properties and behavior. Each 


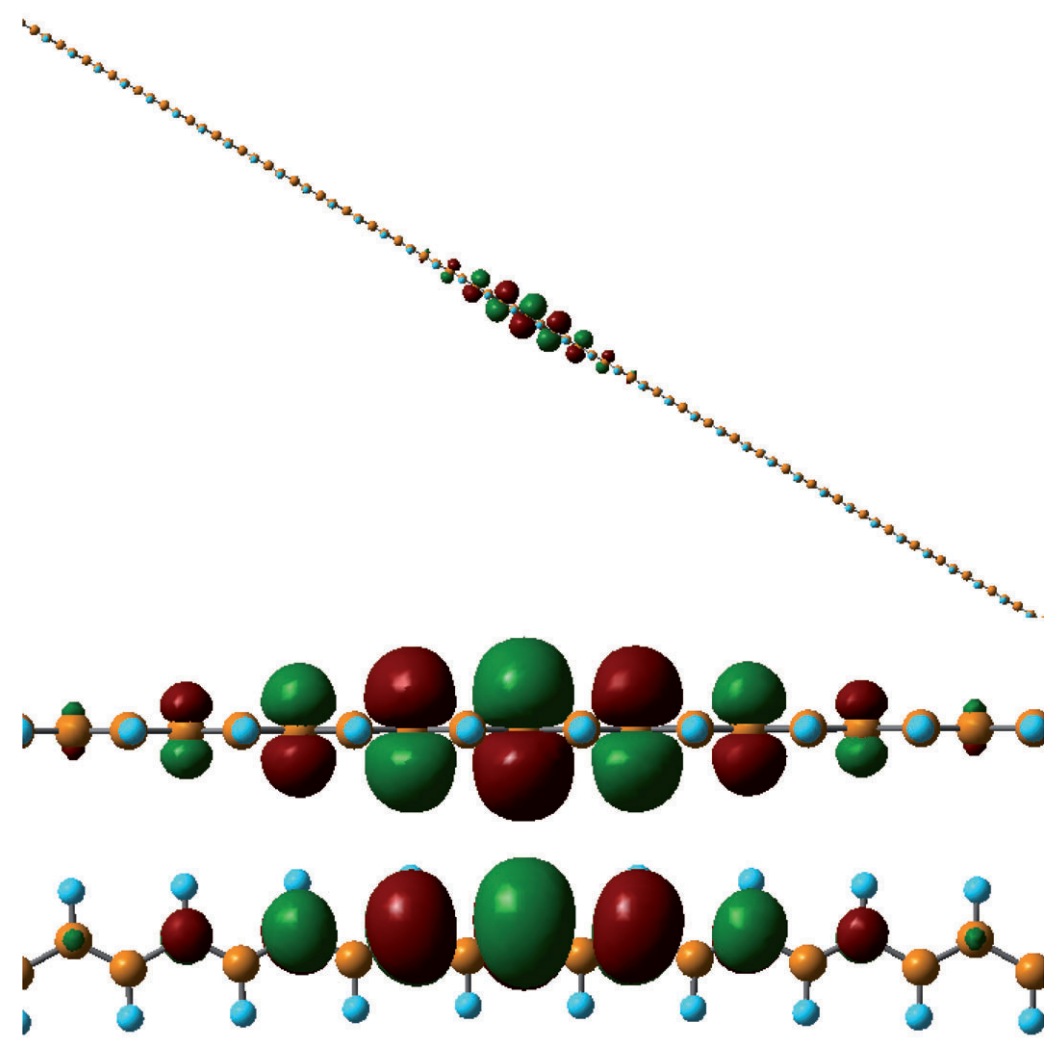

Figure 17. Soliton wavefunction distribution along a $t$-PA chain (a), and its close side (b) and top (c) views, by Hartree-Fock calculations [18]. Orange and blue balls are the carbon and hydrogen atoms, respectively. Wavefunction nodal structures are emphasized by marking the + phases as red lobes and the - phases as green lobes.

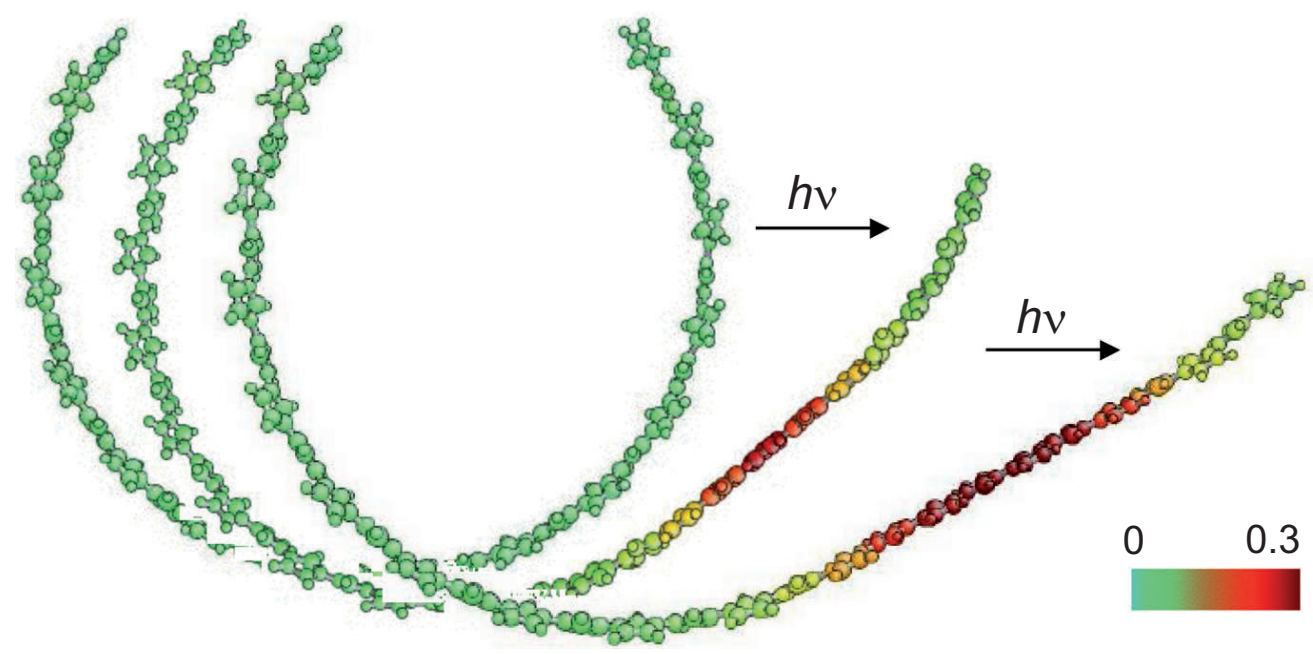

FIGURE 18. Localized charged soliton-anti-soliton pairs, created by photo-excitations or chemical dopings, straighten the polypyrrole chain. Charges are color coded. 


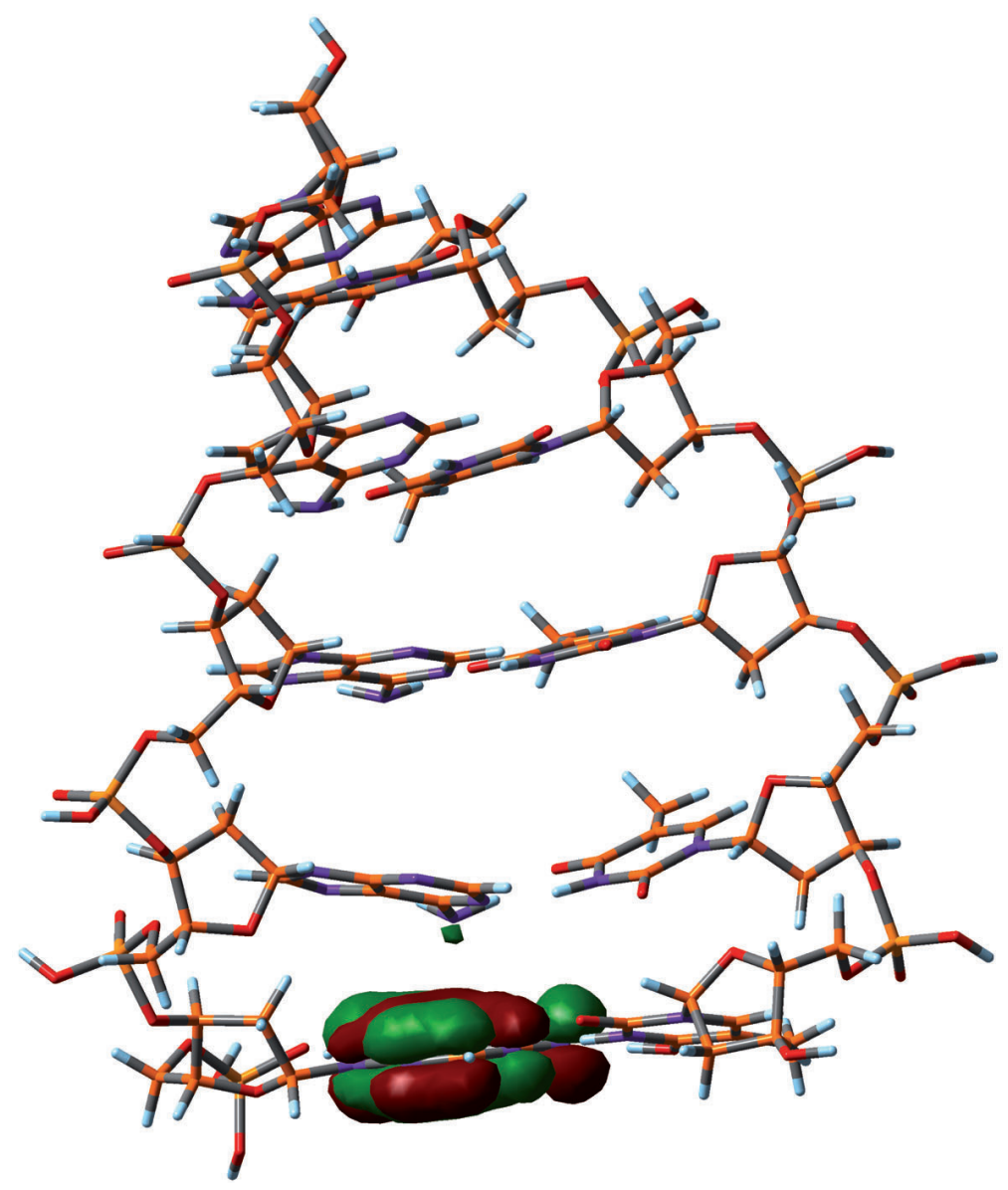

Figure 19. Small polaron (soliton-anti-soliton pair) in poly(dA)-poly(dT) DNA molecule by Hartree-Fock calculations.

problem required a trade-off between the level of modelling accuracy and the length and time scales of the simulation, since more physically accurate models increase the computational cost and are therefore restricted to small length and short time scales. The vision of multiscale materials modelling is to avoid this trade-off by linking the modelling concepts and simulation methods across several length and time scales. In this paradigm electronic structure would be treated by quantum mechanical calculations, atomistic processes by molecular dynamics or Monte Carlo simulations, mesoscale microstructure by methods such as finite-element, dislocation dynamics, or kinetic Monte Carlo, and continuum behavior by field equations well known in fluid and solid mechanics. Comprehensive overviews of the various methods and models that have been developed by the community of multiscale modelling have appeared [9,11,25,34,42,44,57], and more contributions of this type can be expected in the rapidly expanding literature.

There are two major approaches for linking simulation levels, namely sequential and concurrent multiscale methods. In the sequential approach, each level is simulated in isolation, keeping the other scales as fixed inputs. This is easier to implement, since existing codes for each level can be employed with few changes, and it is therefore much more widespread in the literature (e.g. [10]). However, it is better suited to problems where the different levels are weakly coupled, for example due to separate time scales, and can be decoupled on the simulation. The main challenge is to match the inputs of the each scale to the outputs of the others. For example, coupling between continuum and atomistic scales require translation between continuum stresses/strains 
and interatomic forces/positions. Mathematical methodologies and challenges are discussed in references [12] and [13].

The concurrent approach, in which all scales are simulated simultaneously, is more general but much harder to employ, and fewer illustrations can be found in the literature $(e . g .[6,35])$. When the simulation scales are strongly coupled, it is necessary to solve a full set of equations that involve all multiscale degrees of freedom. In most problems, it is possible to decompose the system in domains that require different levels of accuracy. Since each isolated scale is well understood, the main modelling challenge is to obtain the interaction energy between scales, which requires handshaking between the domains, while avoiding the creation of unphysical boundaries. Besides domain partitioning, there are also intrinsically multiscale methods such as the quasicontinuum method [51] and coarse grained molecular dynamics [47], which do not reduce to single scale models but have similar mathematical properties.

Challenges for applied mathematicians arise when distinct physical models are coupled, since the resulting simulations have numerical properties that affect the performance of the solution methods. A simple example is the minimization of the energy of the multiscale system (or finding a saddle point), which becomes a very ill-conditioned problem. A second example is the time integration of the unified set of time evolution equations. The set of ordinary differential equations is extremely stiff, requiring very small time steps to correctly resolve the high frequency vibration modes associated with the smaller scales. This severely limits the length of time that can be simulated, which is already a challenge for atomic-scale methods and becomes critical for multiscale simulations. Rare events are therefore hard to observe, and alternative methods are required, such as reaction pathway sampling [3]. The challenge is to solve, in an efficient and robust way, the variational problem of minimizing the energy of a path in configuration space. The NEB method [23] used in Sections 1.3 and 1.4 has been developed primarily using physical principles. As a consequence, the objective function being minimized is unknown and may not exist [24]. Input from applied mathematicians has provided a new perspective in the form of the string method [14]. This illustrates the benefits of cross fertilization between traditionally different areas of physical science, a development serving the interest of everyone in the community.

\section{REFERENCES}

[1] S.S. Alexandre, E. Artacho, J.M. Soler and H. Chacham, Small polarons in dry DNA. Phys. Rev. Lett. 91 (2003) 108105.

[2] C. Ashman, C. Först, K. Schwarz and P. Blöchl, First-principles calculations of strontium on Si(001). Phys. Rev. B 69 (2004) 075309 .

[3] P.G. Bolhuis, D. Chandler, C. Dellago and P.L. Geissler, Transition path sampling: Throwing ropes over rough mountain passes, in the dark. Annu. Rev. Phys. Chem. 53 (2002) 291-318.

[4] M. Born, On the stability of crystal lattices. I. Proc. Cambridge Philos. Soc. 36 (1940) 160.

[5] M. Born and K. Huang, Dynamical Theory of Crystal Lattices. Clarendon, Oxford (1956).

[6] J.Q. Broughton, F.F. Abraham, N. Bernstein and E. Kaxiras, Concurrent coupling of length scales: Methodology and application. Phys. Rev. B 60 (1999) 2391-2403.

[7] M.J. Buehler, F.F. Abraham and H.J. Gao, Hyperelasticity governs dynamic fracture at a critical length scale. Nature 426 (2003) 141-146.

[8] W. Cai, V.V. Bulatov, J. Chang, J. Li and S. Yip, Dislocation core effects on mobility, in Dislocations in Solids, Vol. 12, Chap. 64, F.R.N. Nabarro and J.P. Hirth Eds., Elsevier, Amsterdam (2004) 1-80.

[9] G.H. Campbell, S.M. Foiles, H.C. Huang, D.A. Hughes, W.E. King, D.H. Lassila, D.J. Nikkel, T.D. De la Rubia, J.Y. Shu and V.P. Smyshlyaev, Multi-scale modeling of polycrystal plasticity: A workshop report. Mater. Sci. Eng. A-Struct. Mater. Prop. Microstruct. Process. 251 (1998) 1-22.

[10] E. Clementi, Global scientific and engineering simulations on scalar, vector and parallel lcap-type supercomputers. Philos. Trans. R. Soc. Lond. Ser. A-Math. Phys. Eng. Sci. 326 (1988) 445-470.

[11] T.D. De la Rubia and V.V. Bulatov, Materials research by means of multiscale computer simulation. Mrs Bull. 26 (2001) $169-175$.

[12] W.E and B. Engquist, The heterogeneous multiscale methods. Comm. Math. Sci. 1 (2003) 87-132.

[13] W.E and B. Engquist, Multiscale modeling and computation. Notices AMS 50 (2003) 1062-1070.

[14] W.N. E, W.Q. Ren and E. Vanden-Eijnden, String method for the study of rare events. Phys. Rev. B 66 (2002) 052301.

[15] R.G. Endres, D.L. Cox and R.R. Singh, The quest for high-conductance DNA. Rev. Mod. Phys. 76 (2004) $195-214$.

[16] C. Först, C. Ashman, K. Schwarz and P. Blöchl, The interface between silicon and a high-k oxide. Nature 427 (2004) 53. 
[17] C. Först, K. Schwarz and P. Blöchl, Structural and electronic properties of the interface between the high-k oxide LaAlO 3 and $\mathrm{Si}(001)$. Phys. Rev. Lett. 95 (2005) 137602.

[18] M.J. Frisch et al. Gaussian 03. Gaussian, Inc. (2003).

[19] A. Gouldstone, H.J. Koh, K.Y. Zeng, A.E. Giannakopoulos and S. Suresh, Discrete and continuous deformation during nanoindentation of thin films. Acta Mater. 48 (2000) 2277-2295.

[20] A.J. Heeger, S. Kivelson, J.R. Schrieffer and W.-P. Su, Solitons in conducting polymers. Rev. Mod. Phys. 60 (1988) $781-851$.

[21] R. Hill, Acceleration waves in solids. J. Mech. Phys. Solids 10 (1962) 1-16.

[22] International technology roadmap for semiconductors (2003). Available at http://public.itrs.net/

[23] H. Jonsson, G. Mills and K.W. Jacobsen, Nudged elastic band method for finding minimum energy paths of transitions, in Classical and Quantum Dynamics in Condensed Phase Simulations, B.J. Berne, G. Ciccotti and D.F. Coker Eds., World Scientific (1998) 385-404.

[24] H. Jónsson, G. Mills and K.W. Jacobsen, Nudged elastic band method for finding minimum energy paths of transitions, in Classical and Quantum Dynamics in Condensed Phase Simulations, Chap. 16, B.J. Berne, G. Ciccotti and D.F. Coker. Eds., World Scientific (1998) 385-404.

[25] E. Kaxiras and S. Yip, Modelling and simulation of solids - Editorial overview. Curr. Opin. Solid State Mat. Sci. 3 (1998) 523-525.

[26] A.E. Leanhardt, T.A. Pasquini, M. Saba, A. Schirotzek, Y. Shin, D. Kielpinski, D.E. Pritchard and W. Ketterle, Cooling bose-einstein condensates below 500 picokelvin. Science 301 (2003) 1513-1515.

[27] J. Li, K.J.V. Vliet, T. Zhu, S. Yip and S. Suresh, Atomistic mechanisms governing elastic limit and incipient plasticity in crystals. Nature 418 (2002) 307-310.

[28] J. Li, T. Zhu, S. Yip, K.J.V. Vliet and S. Suresh, Elastic criterion for dislocation nucleation. Mater. Sci. Eng. A-Struct. Mater. Prop. Microstruct. Process. 365 (2004) 25-30.

[29] M.J. Lii, X.F. Chen, Y. Katz and W.W. Gerberich, Dislocation modeling and acoustic-emission observation of alternating ductile/brittle events in Fe-3wt\% Si crystals. Acta Metall. Mater. 38 (1990) 2435.

[30] X. Lin, J. Li and S. Yip, Controlling bending and twisting of conjugated polymers via solitons. Phys. Rev. Lett. 95 (2005) 198303.

[31] X. Lin, J. Li, E. Smela and S. Yip, Polaron-induced conformation change of a single polypyrrole chain: An intrinsic actuation mechanism. Int. J. Quant. Chem. 102 (2005) 980-985.

[32] X. Lin, J. Li, C. Foerst and S. Yip, Multiple self-localized electronic states in trans-polyacetylene. Proc. Natl. Acad. Sci. 103 (2006) 8943-8946.

[33] W.A. Little, Possibility of synthesizing an organic superconductor. Phys. Rev. 134 (1964) A1416-A1424.

[34] G. Lu and E. Kaxiras, Overview of multiscale simulation of materials, in Handbook of Theoretical and Computational Nanotechnology, Vol. X, M. Rieth and W. Schommers Eds., American Scientific Publ. (2005) 1-33.

[35] G. Lu, E.B. Tadmor and E. Kaxiras, From electrons to finite elements: A concurrent multiscale approach for metals. Phys. Rev. B $\mathbf{7 3}$ (2006) 024108.

[36] A.G. MacDiarmid, "Synthetic metals": A novel role for organic polymers. Rev. Mod. Phys. 73 (2001) 701-712.

[37] R. McKee, F. Walker and M. Chisholm, Crystalline oxides on silicon: the first five monolayers. Phys. Rev. Lett. 81 (1998) 3014.

[38] T.A. Michalske and S.W. Freiman, A molecular interpretation of stress-corrosion in silica. Nature 295 (1982) 511-512.

[39] Y. Mishin, M.J. Mehl, D.A. Papaconstantopoulos, A.F. Voter and J.D. Kress, Structural stability and lattice defects in copper: Ab initio, tight-binding and embedded-atom calculations. Phys. Rev. B 6322 (2001) 224106.

[40] J.W. Morris and C.R. Krenn, The internal stability of an elastic solid. Philos. Mag. A 80 (2000) 2827-2840.

[41] S. Ogata, J. Li, N. Hirosaki, Y. Shibutani and S. Yip, Ideal shear strain of metals and ceramics. Phys. Rev. B 70 (2004) 104104.

[42] M. Ortiz and R. Phillips, Nanomechanics of defects in solids. Advan. Appl. Mech. 36 (1999) 1-79.

[43] R.E. Peierls, Quantum Theory of Solids. The International series of monographs on physics. Oxford University Press, New York (1955).

[44] R. Phillips, Multiscale modeling in the mechanics of materials. Curr. Opin. Solid State Mat. Sci. 3 (1998) 526-532.

[45] J.R. Rice, in Theoretical and Applied Mechanics, Vol. 1., W.T. Koiter Ed., North-Holland, Amsterdam (1976) 207.

[46] S. Roth and D. Carroll, One-Dimensional Metals. Wiley-VCH, Weinheim, 2nd edn. (2004).

[47] R.E. Rudd and J.Q. Broughton, Coarse-grained molecular dynamics and the atomic limit of finite elements. Phys. Rev. B 58 (1998) R5893-R5896.

[48] J.J.P. Stewart, MOPAC 2002 Manual. Fujitsu Ltd., Tokyo (2002).

[49] F.H. Stillinger and T.A. Weber, Computer-simulation of local order in condensed phases of silicon. Phys. Rev. B 31 (1985) $5262-5271$

[50] W.P. Su, J.R. Schrieffer and A.J. Heeger, Solitons in polyacetylene. Phys. Rev. Lett. 42 (1979) 1698-1701.

[51] E.B. Tadmor, M. Ortiz and R. Phillips, Quasicontinuum analysis of defects in solids. Philos. Mag. A-Phys. Condens. Matter Struct. Defect Mech. Prop. 73 (1996) 1529-1563.

[52] K.J.V. Vliet, J. Li, T. Zhu, S. Yip and S. Suresh, Quantifying the early stages of plasticity through nanoscale experiments and simulations. Phys. Rev. B $6 \mathbf{7}$ (2003) 104105. 
[53] D.C. Wallace, Thermodynamics of Crystals. Wiley, New York (1972).

[54] J.H. Wang, J. Li, S. Yip, S. Phillpot and D. Wolf, Mechanical instabilities of homogeneous crystals. Phys. Rev. B 52 (1995) 12627-12635.

[55] J.H. Wang, J. Li, S. Yip, D. Wolf and S. Phillpot, Unifying two criteria of Born: Elastic instability and melting of homogeneous crystals. Physica A 240 (1997) 396-403.

[56] S.M. Wiederhorn, Fracture surface energy of glass. J. Am. Ceram. Soc. 52 (1969) 99-105.

[57] S. Yip Ed., Handbook of Materials Modeling. Springer, Dordrecht (2005).

[58] Y. Yu, M. Nakano and T. Ikeda, Directed bending of a polymer film by light. Nature 425 (2003) 145.

[59] Z. Zhou and B. Joos, Stability criteria for homogeneously stressed materials and the calculation of elastic constants. Phys. Rev. B 54 (1996) 3841-3850.

[60] T. Zhu, J. Li and S. Yip, Atomistic study of dislocation loop emission from a crack tip. Phys. Rev. Lett. 93 (2004) 025503.

[61] T. Zhu, J. Li and S. Yip, Atomistic configurations and energetics of crack extension in silicon. Phys. Rev. Lett. 93 (2004) 205504. 